\title{
The Impact of COVID-19 on Gastric Cancer Surgery: A Single-center Retrospective Study Based on Real- world Data
}

\section{Yu-xuan Li}

Chinese PLA General Hospital

Chang-zheng He

Chinese PLA General Hospital

Yi-chen Liu

Chinese PLA General Hospital

Peng-yue Zhao

Chinese PLA General Hospital

Xiao-lei Xu

Chinese PLA General Hospital

Yu-feng Wang

Chinese PLA General Hospital

Shao-you Xia

Chinese PLA General Hospital

Xiao-hui Du ( $\sim$ duxiaohui301@sina.com )

\section{Research}

Keywords: gastric cancer; coronavirus disease 2019; COVID-19; retrospective analy-sis; real-world data.

Posted Date: April 28th, 2020

DOI: https://doi.org/10.21203/rs.3.rs-22785/v1

License: (c) (i) This work is licensed under a Creative Commons Attribution 4.0 International License.

Read Full License 


\title{
The Impact of COVID-19 on Gastric Cancer Surgery: A Single-center Retrospec- tive Study Based on Real-world Data
}

\author{
Yu-xuan Li ${ }^{1}$,, Chang-zheng He ${ }^{1, \#}$, Yi-chen Liu ${ }^{1, \#}$, Peng-yue Zhao ${ }^{1}$, Xiao-lei Xu ${ }^{1}$, \\ Yu-feng Wang ${ }^{2}$, Shaoyou Xia ${ }^{1}$, Xiao-hui Du ${ }^{1}$
}

${ }^{1}$ Department of General Surgery, Chinese General Hospital of People's Liberation Army, Beijing 100853, PR China

${ }^{2}$ Department of Hospitalization management, Chinese General Hospital of People's Liberation Army, Beijing 100853, PR China

\section{Corresponding Authors:}

Xiao-hui Du, MD, PhD, Department of General Surgery, Chinese General Hospital of People's Liberation Army, Beijing 100853, PR China. Tel: (+86) 13801300826; Email:duxiaohui301@sina.com. Shao-you Xia, MD, phD, Department of General Surgery, Chinese General Hospital of People's Liberation Army, Beijing 100853, PR China. Tel: (+86) 13301311136; Email:lyx1389@126.com

\# These authors contributed equally to this manuscript. 


\section{Abstract}

Background: A respiratory epidemic defined as coronavirus disease 2019 (COVID19) is becoming unstoppable and has been declared a pandemic. Patients with cancer are more likely to develop COVID-19. Based on our experience during the pandemic period, we propose some surgery strategies for gastric cancer patients under the COVID-19 situation.

Methods: We defined the 'COVID-19' period as occurring between 2020-01-20 and 2020-03-20. All the enrolled patients were divided into two groups, pre-COVID-19 group (PCG) and COVID-19 group (CG). A total of 109 patients with gastric cancer were enrolled in this study.

Results : The waiting times before admission increased by 4 days in CG(PCG:4.5 [IQR: 2, 7.8] vs. CG:8.0 [IQR: 2,20]; $\mathrm{P}=0.006$ ). More patients had performed chest $\mathrm{CT}$ scan besides abdominal CT before admission during COVID-19 period(PCG:22[32\%]vs. $\mathrm{CG}: 30[73 \%], \mathrm{p}=0.001)$. After admission, during COVID period, the waiting time before surgery was longer(3[IQR: 2,5] vs. 7[IQR: 5,9]; P $<0.001)$,more laparoscopic surgery were performed(PCG: $51[75 \%]$ vs. CG: 38[92\%],p=0.021), and hospital stay after surgery was longer (7[IQR: 6,8] vs.9[IQR:7,11] ; $\mathrm{P}<0.001)$. The total cost of hospitalization increased during COVID period, (9.22[IQR:7.82,10.97] vs. 10.42[IQR:8.99,12.57]; $\mathrm{p}=0.006)$.

Conclusion : Since no data is available yet on the impact of COVID-19 on gastric cancer patients, our own experience with COVID-19 in gastric cancer surgery has hopefully provided an opportunity for colleagues to reflect on their own service and any contingency plans they have to tackle the crisis.

Keywords: gastric cancer; coronavirus disease 2019; COVID-19; retrospective analysis; real-world data. 


\section{Background}

A respiratory epidemic defined as coronavirus disease 2019 (COVID-19) emerged in Wuhan city, Hubei Province, China in late December 2019. 41 patients were admitted to hospitals with an initial diagnosis of pneumonia of an unknown etiology, most of the them had visited a local fish and wild animal market in November $(1,2)$. Then the spread of COVID-19 is becoming unstoppable and has been declared a pandemic. According to the Situation Report-77 of WHO (Data as of 6 April 2020), more than 1.2 million people have been infected all over the world (3).

Although most patients presented with symptoms such as fever, dry cough and dyspnea, the incidence of isolated or coexisting abdominal and gastrointestinal symptoms such as diarrhea, nausea, vomiting and abdominal discomfort were also common(4), which resulted in the implementation of stringent precautionary measures for patients and particularly hospital staff who were at greatest risk from the contaminated droplets and bodily fluids of the patients $(5,6)$.

Gastric cancer surgery is not a front-line subject in the fight against the COVID-19, however, in such a special situation, patients with cancer are more likely to develop COVID-19 $(7,8)$. As the virus can be transmitted by asymptomatic infectors, and it has been confirmed to be transmitted by droplets and contact (9), it is important to protect the safety of surgeons, nurses, patients and their families from infection (10).

Based on our experience during the pandemic period, we propose some surgery strategies for gastric cancer patients under the COVID-19 situation.

\section{Methods}

\section{Study Design and Patients}

The General Surgery Department of our hospital serves as a final referral unit for a cluster of hospitals from other districts and provides specialist services for gastric cancer. On January 20, 2020, National Health Commission of the PRC announced that 
pneumonia infected by the new coronavirus would be included in the management of the statutory infectious disease category B, but the prevention and control measures for the infectious disease were category .Since then we've applied intensified strategies and managements composed of three aspects of outpatients, inpatients and discharged patients, in case of COVID-19 infection On March 20, 2020,Beijing,the city our hospital locates, had no new local confirmed COVID-19 case for consecutive days, which was a milestone in the battle against the virus. Based on the above, we defined the 'COVID-19' period as occurring between 2020-01-20 and 2020-03-20. Data for this period was compared with a similar preceding 1-month period between 2020-12-20 and 2020-1-19 which we termed 'Pre-COVID-19'. Thus all the enrolled patients were divided into two groups, pre-COVID-19 group (PCG) and COVID-19 group (CG), based on which period they were admitted to our hospital.

All of the case series was approved by the Institutional Review Board of General Hospital of PLA. Inclusion criteria were as follows: (1) Gastric cancer patients were diagnosis based on pathological examination and all those clinical data were integral and available. (2) All the patients received surgery treatment. (3) Patients who received neoadjuvant chemotherapy (NACT) or underwent emergency surgery were excluded. A total of 109 patients with gastric cancer enrolled in this study were diagnosed according to The NCCN Clinical Practice Guidelines in Oncology (NCCN Guidelines) for Gastric Cancer. Operations were performed by the same team of surgeons.

\section{Data Collection}

The medical records of patients were collected and analyzed by our research team. We summarized the clinical, epidemiological, radiological ,laboratory characteristics from electronic medical records, including patient demographics and baseline characteristics(sex, age, body mass index, comorbidity, clinical TNM classification, pathological TNM classification, chemotherapy regimen, hemoglobin, neutrophil-to-lymphocyte 
ratio,CEA,CA199,AFP,CA724),surgical procedure, operating time, estimated blood loss ,postoperative complications ,postoperative body temperature, waiting times for the admission ,length of postoperative hospital stay ,hospital costs etc.

\section{Statistical Analysis}

SPSS version 26.0 was used for statistical analysis. Normality assumption for all quantitative variables data was verified with the Shapiro-Wilk test. Data were expressed as mean \pm standard deviation $(\overline{x \pm s})$ for normal distribution variables.For the data that did not conform to the normal distribution, the median and interquartile range were used to express the measurement data , and count data were expressed by frequency and percentage (\%). Student $t$ tests were applied to compare means for normally distributed variables and Mann-Whitney test for variables without normal distribution. Statistical analysis of count data using chi-square test or Fisher exact probability method.

\section{Results}

\section{Study population and baseline demographics}

Between December 20,2019 to March 20,2020,a total of 109 patients were enrolled in this study. For the 109 patients who underwent elective surgery, 68 of them were enrolled into PCG and 41 were into CG(Figure 1). Patients demographics for PCG and CG were shown in Table 1. The was no difference between PCG and CG in age, sex, Body mass index $(\mathrm{P}>0.05)$.

\section{Clinicopathological data of all patients enrolled before or after $20^{\text {th }}$ January}

Pre-COVID-19, admissions to the gastric unit totaled 68 cases over the 1-month period. During COVID-19, 2-months period, the total dropped by 30\% to 41 admissions. In contrast, the waiting times before admission increased by 4 days (PCG:4.5 [IQR: 2, 7.8] vs. CG:8.0 [IQR: 2,20]; $\mathrm{P}=0.006$ ) . The proportion of local patients in 
PCG is lower than in CG (3 [4\%] vs. 16 [39\%]; P < 0.001). More patients had performed chest CT scan besides abdominal CT before admission during COVID-19 period (PCG:22[32\%] vs. CG:30[73\%], p=0.001). The granulolymphatic ratio in $\mathrm{CG}$ was lower than in PCG, $(2.83 \pm 3.21$ vs. $1.90 \pm 1.08 ; \mathrm{p}=0.03)$. (Table 2)

After admission, during COVID-19 period, the waiting time before surgery was longer (3[IQR: 2,5] vs. 7[IQR: 5,9]; P < 0.001),more laparoscopic surgery were performed (PCG: 51[75\%] vs. CG: 38[92\%],p=0.021), and hospital stay after surgery was longer (7[IQR: 6,8] vs.9[IQR:7,11] ; P $<0.001)$. There was no statistical difference in surgical time, pathological diagnosis, TNM staging, complication including pneumonia and blood transfusion, as well as highest temperature, screening test and reason for fever between two groups $(\mathrm{P}>0.05)$. (Table 3-4)

\section{Health economics data of all patients enrolled before or after $20^{\text {th }}$ January}

The total cost of hospitalization increased during COVID period, (9.22[IQR:7.82,10.97] vs. 10.42[IQR:8.99,12.57]; $\mathrm{p}=0.006)$, Medicine cost, treatment cost and other aspects including beds, cares, diets etc. were higher in $\mathrm{GC}(\mathrm{p}>0.05)$. The results were shown in Table 5.

\section{Discussion}

The COVID-19 outbreak alerted us the importance of infection control measures for pandemic diseases. Successful implementation of infection control required strict management of inpatients during this period. Due to disease consumption, malnutrition, coupled with chemotherapy, gastric cancer patients may be immunocompromised, which leads to more susceptible to COVID-19 $(4,8)$.Therefore, we suggest that all outpatients should be triaged before admission to reduce the possibility of exposure in hospital. Making personalized diagnosis and treatment plans for gastric cancer patients by multidisciplinary team (MDT) are essential $(11,12)$. departments and staff members of MDT include: general surgery, oncology, radiology, radiotherapy, respiratory medicine, and infectious disease experts. It is recommended that patients 
should have completed gastroscopy, pathological examinations and imaging examinations (abdominal, pelvic CT or MRI, including PET-CT if necessary) at the local hospital before visiting our clinic. The MDT clinic comprehensively evaluates the gastric cancer patients based on clinical symptoms, pathological classification, imaging staging, and other examination, which resulted in precisely determination of the cTNM stage.

EMR or ESD are suggested for patients with early-stage gastric cancer lesions $\leq 2$ $\mathrm{cm}$ in diameter without associated ulcer formation (cTis or cT1a). Surgery is the primary treatment option for $\mathrm{cT} 1 \mathrm{~b}$ patients. And patients with histologically confirmed gastric adenocarcinoma of cT2 to cT4a were suggested to receive perioperative chemotherapy. This shunt method allows patients to receive appropriate treatment, while reducing the number of hospitalized patients during the pandemic and reducing the risk of infection. Especially for patients who have finished neoadjuvant chemotherapy in several month later, they could benefit from avoiding the peak of the pandemic. However, for resectable gastric cancer patients with anemia, refusal of neoadjuvant chemotherapy, or low granulolymphatic ratio, delaying the time of radical surgery will not benefit the patient. Therefore, timely surgery might be a better choice. During the 'COVID-19' period, we recommend chest CT and new coronavirus nucleic acid tests to rule out patients with new coronary pneumonia and suspicious infection, which explains the longer waiting time before admission and higher chest $\mathrm{CT}$ scan rates in $\mathrm{CG}$ compared to PCG. In addition, the provinces were relatively isolated during the pandemic, therefore, compared with PCG, the proportion of local patients in CG had increased.

After admission, patients were isolated in separated single-room wards without contact to surgeons or nurses, which helps us to observe whether they manifested elevated temperature or symptoms suspicious for COVID-19. If the patients did not had a fever of over $37.3^{\circ} \mathrm{C}$ or other symptoms associated to pneumonia for 3 days since admission, the surgery would then be performed. During the pandemic, routine 
surgery should be based on the principles of safety and efficiency, with the main purpose of reducing the incidence of postoperative complications and accelerating the patient's recovery and discharge $(12,13)$. Therefore, it is necessary to avoid performing surgery beyond authoritative guidelines, including oversized lymph node dissections with uncertain effects and complex digestive tract reconstruction methods. Attention should be paid to clear anatomy and accurate operation during surgery to reduce the risk of bleeding during and after surgery due to the tight blood source in the blood bank during the pandemic period, which could decrease unexpected blood transfusion treatment.

Enhanced Recovery After Surgery(ERAS) pathway was initially established by Henrik Kehlet (14) in colorectal surgery, Since then the idea has gradually been accepted .However, the first ERAS guideline for gastric cancer were published in 2014, and most evidence of it based on western countries. Lacking of gastric cancer ERAS protocol appropriate to Chinese conditions hampered us from accepting it at the first time. However, in recent years we are gradually conducting clinical trials on ERAS for gastric cancer. In the light of COVID- 19 pandemic, ERAS for gastric cancer patients demonstrated considerable advantages since it could reduce the patient's surgical stress response, postoperative complications, and shorten the length of hospital stay, thereby reducing the chance of nosocomial infection. While, the total hospital stay of patients in CG was longer than that in PCG, which seems ERAS was not applied to patients in CG. However, considering that during the COVID-19 period, patients had to remove the stitches in outpatient clinics and local hospitals since discharged, which would increase the risk of unnecessary viral infections, It was better to stay longer in our department until stitches were removed. In addition, other ERAS procedures in the two periods were almost analogous. 
After surgery, If the patient had fever of unknown cause, appropriate ward isolation measures should be taken and postoperative blood routine, C-reactive protein, procalcitonin, chest $\mathrm{CT}$, and new coronavirus nucleic acid tests are necessary.

Perioperative chemotherapy has significantly increased survival in patients with locoregional resectable gastric cancer. Capecitabine combined with Oxaliplatin or S-1 (SOX or XELOX), has been applied as one of the standard perioperative chemotherapy strategy for patients with advanced gastric cancer in Chinese population (1518). The preoperative chemotherapy schedules were composed of Oxaliplatin (130 $\mathrm{mg} / \mathrm{m}^{2}$ intravenously on day 1 every 3 weeks), combined with S-1 (40/50/60 mg orally twice daily for 14 days followed by 7 days off, The does depends on surface area: $\left.<1.25 \mathrm{~m}^{2} / 1.25-1.5 \mathrm{~m}^{2} />1.5 \mathrm{~m}^{2}\right)(\mathrm{SOX})$ or Capecitabine $(1000 \mathrm{mg} / \mathrm{m} 2$ twice daily for 14 days followed by 7 days off) (XELOX). Patients were admitted to daycare ward for intravenous injection of Oxaliplatin every 3 weeks, which shorten patient exposure time in hospital due to surgical treatment and reduce the risk of cross infection. The radical gastrectomy surgery would be performed in 4 weeks after $2-4$ cycles neoadjuvant chemotherapy, at that time, the peak of the pandemic might pass.

\section{Conclusions}

In conclusion, no data is available yet on the impact of COVID-19 (if any) on gastric cancer patients. The full impact of COVID-19 on surgery is still unknown, since its effects on global economics, politics, hospital management, health strategy and personnel may only become evident in the long term. Our own experience with COVID-19 in gastric cancer surgery has hopefully provided an opportunity for colleagues to reflect on their own service and any contingency plans they have to tackle the crisis. 


\section{Ethics approval and consent to participate}

All of the case series was approved by the Institutional Review Board of General Hospital of PLA(S2020-003-01). The need to obtain informed consent was waived because of its retrospective nature. In our hospital, every patient have right to refuse the use of their record at any time.

\section{Consent for publication}

Not applicable.

\section{Availability of data and materials}

All data generated or analysed during this study are included in this published article.

\section{Competing interests}

The authors declared no competing interests.

\section{Funding}

This work was supported by grants from the National Natural Science Foundation of China (No. 81871317)

\section{Authors' contributions}

XHD and SYX conceived the analysis. YXL, CZH and YCL extracted all data. YFW, PYZ and XLX undertook and refined the inclusion process. YXL, CZH and YCL co-wrote the paper. YXL, YCL and YFW undertook the statistical analyses. All authors contributed to and revised the final manuscript.

\section{Acknowledgements}

Not applicable. 


\section{Reference}

1.Lu, H., C.W. Stratton, and Y.W. Tang, Outbreak of pneumonia of unknown etiology in Wuhan, China: The mystery and the miracle. J Med Virol, 2020. 92(4): p. 401-402. 2. Rothan, H.A. and S.N. Byrareddy, The epidemiology and pathogenesis of coronavirus disease (COVID-19) outbreak. J Autoimmun, 2020: p. 102433.

3. Remuzzi, A. and G. Remuzzi, COVID-19 and Italy: what next? The Lancet, 2020. 4. Wang, D., et al., Clinical Characteristics of 138 Hospitalized Patients With 2019 Novel Coronavirus-Infected Pneumonia in Wuhan, China. JAMA, 2020.

5. Danese, S., M. Cecconi, and A. Spinelli, Management of IBD during the COVID19 outbreak: resetting clinical priorities. Nat Rev Gastroenterol Hepatol, 2020.

6. Gagliano, A., et al., 2019-ncov's epidemic in middle province of northern Italy: impact, logistic \& strategy in the first line hospital. Disaster Med Public Health Prep, 2020: p. 1-15.

7. Pellino, G. and A. Spinelli, How COVID-19 Outbreak Is Impacting Colorectal Cancer Patients in Italy: A Long Shadow Beyond Infection. Dis Colon Rectum, 2020.

8. Wang, H. and L. Zhang, Risk of COVID-19 for patients with cancer. The Lancet Oncology, 2020. 21(4).

9. Gu, J., B. Han, and J. Wang, COVID-19: Gastrointestinal manifestations and potential fecal-oral transmission. Gastroenterology, 2020.

10. Tan, Y.M., et al., Management of inpatients exposed to an outbreak of severe acute respiratory syndrome (SARS). J Hosp Infect, 2004. 58(3): p. 210-5.

11. Chen, Y.H. and J.S. Peng, [Treatment strategy for gastrointestinal tumor under the outbreak of novel coronavirus pneumonia in China]. Zhonghua Wei Chang Wai Ke Za Zhi, 2020. 23(2): p. I-IV.

12. Hu, X.H., et al., [Treatment strategies for colorectal cancer patients in tumor hospitals under the background of corona virus disease 2019]. Zhonghua Wei Chang Wai Ke Za Zhi, 2020. 23(3): p. 201-208.

13. Ma, F.H., H.T. Hu, and Y.T. Tian, [Surgical treatment strategy for digestive system malignancies during the outbreak of novel coronavirus pneumonia]. Zhonghua Zhong Liu Za Zhi, 2020. 42(0): p. E001. 
14. Bardram L, Funch-Jensen P, Jensen P, et al. Recovery after laparoscopic colonic surgery with epidural analgesia, and early oral nutrition and mobilisation[J]. Lancet (North American Edition), 1995, 345(8952):763-764.

15. Cunningham D, Allum W H, Stenning S P, et al. Perioperative Chemotherapy versus Surgery Alone for Resectable Gastroesophageal Cancer[J]. The Journal of Evidence-Based Medicine, 2008, 355(1):11.

16. Li T, Chen L . [Efficacy and safety of SOX regimen as neoadjuvant chemotherapy for advanced gastric cancer][J]. Chinese Journal of Gastrointestinal Surgery, 2011, 14(2):104-106.

17. Cunningham D, Starling N, Rao S, et al. Capecitabine and Oxaliplatin for Advanced Esophagogastric Cancer[J]. New England Journal of Medicine, 2008, 358(1):36-46.

18.Wang, F.H., et al., The Chinese Society of Clinical Oncology (CSCO): clinical guidelines for the diagnosis and treatment of gastric cancer. Cancer Commun (Lond), 2019. 39(1): p. 10. 
Table 1. Baseline demographical data of all patients enrolled

\begin{tabular}{lccc}
\hline \multicolumn{1}{c}{ Demographics } & $\begin{array}{c}\text { PCG }(\mathrm{N}=68) \\
\mathrm{M} \pm \mathrm{SD} \text { or N }(\%)\end{array}$ & $\begin{array}{c}\mathrm{CG}(\mathrm{N}=41) \\
\mathrm{M} \pm \mathrm{SD} \text { or N }(\%)\end{array}$ & $P$ \\
\hline Age (years) & $59.60 \pm 11.04$ & $58.41 \pm 10.27$ & 0.578 \\
Sex & & & 0.583 \\
Male & 45 & 25 & \\
Female & 23 & 16 & 0.099 \\
BMI & $24.37 \pm 3.68$ & $23.30 \pm 2.91$ & $0.000^{*}$ \\
Origin of patients & & & \\
Local patients & 3 & 16 & \\
Other provinces & 65 & 25 & \\
\hline
\end{tabular}

PCG: pre-COVID-19 group; CG: COVID-19 group; M: mean; SD: standard deviation; $\mathrm{N}$ : number.

$* P<0.05$, statistically different 
Table 2. Preoperative clinicopathological data of all patients enrolled

\begin{tabular}{|c|c|c|c|}
\hline \multirow{2}{*}{ Clinicopathologic data } & $\operatorname{PCG}(\mathrm{N}=68)$ & $\mathrm{CG}(\mathrm{N}=41)$ & \multirow{2}{*}{$P$} \\
\hline & $\mathrm{M}(\mathrm{IQR}) \operatorname{orN}(\%)$ & M(IQR)orN (\%) & \\
\hline Admission waiting(day) & $4.5(2-7.75)$ & $8.0(2-20)$ & $0.006^{*}$ \\
\hline Operation waiting(day) & $3(2-5)$ & $7(5-9)$ & $0.000^{*}$ \\
\hline Comorbidity & & & 0.144 \\
\hline Yes & 46 & 22 & \\
\hline No & 22 & 19 & \\
\hline \multicolumn{4}{|l|}{ Tumor marker } \\
\hline CEA & 2.12 & 1.85 & 0.298 \\
\hline CN & 2.85 & 3.01 & 0.771 \\
\hline C100 & 9.70 & 9.63 & 0.883 \\
\hline CA199 & 2.03 & 2.13 & 0.863 \\
\hline CA724 & & & \\
\hline Clinical TNM stage & & & 0.112 \\
\hline I & 17 & 4 & \\
\hline II & 18 & 14 & \\
\hline III & 30 & 23 & \\
\hline IV & 3 & 0 & \\
\hline NLR & $1.78(1.80-2.60)$ & $1.63(1.24-2.23)$ & 0.423 \\
\hline Hemoglobin & $138.50(119.25-148.75)$ & $\begin{array}{l}143.00(119.50- \\
151.00)\end{array}$ & 0.726 \\
\hline Chest CT scan & & & $0.000 *$ \\
\hline Yes & 24 & 30 & \\
\hline No & 44 & 11 & \\
\hline
\end{tabular}

PCG: pre-COVID-19 group; CG: COVID-19 group;NLR: Neutrophilto-Lymphcyte ratio CT: computed tomography; c; N: number.

$* P<0.05$, statistically different 
Table 3. Intraoperative clinicopathological data of all patients enrolled

\begin{tabular}{lccc}
\hline Clinicopathologic data & PCG (N=68) & CG (N=41) & $P$ \\
\hline Surgery time (min) & $184.74 \pm 44.36$ & $195.00 \pm 57.49$ & 0.298 \\
Estimated blood loss (ml)\# & $100(50-137)$ & $100(50-150)$ & 0.569 \\
Operative method & 17 or N (\%) & M SD or N (\%) & $0.021^{*}$ \\
Open & 51 & 3 & \\
Laparoscopic & & 38 & $0.000^{*}$ \\
Combine organ resection & 1 & 0 & \\
Yes & 67 & 41 & \\
No & & & \\
Transfusion of blood & 4 & 6 & \\
Yes & 64 & 35 & \\
No & & & \\
\hline
\end{tabular}

PCG: pre-COVID-19 group; CG: COVID-19 group; M: mean; SD: standard deviation;

$\mathrm{N}$ : number. \# M: median; IQR: inter-quartile range

* $P<0.05$, statistically different 
Table 4. Postoperative clinicopathological data of all patients enrolled

\begin{tabular}{|c|c|c|c|}
\hline Clinicopathologic data & $\begin{array}{r}\mathrm{PCG}(\mathrm{N}=68) \\
\mathrm{M}(\mathrm{IQR}) \operatorname{orN}(\%)\end{array}$ & $\begin{array}{c}\mathrm{CG}(\mathrm{N}=41) \\
\mathrm{M}(\mathrm{IQR}) \operatorname{orN}(\%)\end{array}$ & $P$ \\
\hline Pathological TNM staging & & & 0.394 \\
\hline I & 18 & 8 & \\
\hline II & 18 & 10 & \\
\hline III & 29 & 23 & \\
\hline IV & 3 & 0 & \\
\hline Complications & & & 0.081 \\
\hline Yes & 6 & 0 & \\
\hline No & 62 & 41 & \\
\hline Postoperative fever & & & 0.379 \\
\hline Yes & 29 & 14 & \\
\hline No & 39 & 27 & \\
\hline Highest temperature $\left({ }^{\circ} \mathrm{C}\right)$ & & & 0.587 \\
\hline$<37.3$ & 39 & 27 & \\
\hline $37.3-38.5$ & 22 & 12 & \\
\hline$>38.5$ & 7 & 2 & \\
\hline Screening examination & & & $0.012 *$ \\
\hline None & 16 & 2 & \\
\hline $\mathrm{BRE}+\mathrm{CRP}$ & 8 & 4 & \\
\hline $\mathrm{BRE}+\mathrm{CRP}+\mathrm{CT}$ & 4 & 5 & \\
\hline Nucleic acid test & 0 & 2 & \\
\hline Reason of fever & & & 0.423 \\
\hline Abdominal infection & 1 & 0 & \\
\hline Pulmonary infection & 1 & 0 & \\
\hline Incision infection & 4 & 0 & \\
\hline Anastomotic leakage & 1 & 0 & \\
\hline Unclear & 17 & 11 & \\
\hline
\end{tabular}


None

Transfusion of blood

Yes

No

Postoperative hospital

stay (day)

Total hospital stay (day)

$11(9-13)$

65

$$
7(6-8)
$$

3

0.422

4

37

$9(7-11)$

$0.000 *$

PCG: pre-COVID-19 group; CG: COVID-19 group; BRE: blood routine examination;

CRP: C-reactive protein; CT: computed tomography; Postoperative fever $\left(\geq 37.3^{\circ} \mathrm{C}\right)$

M: median; IQR: inter-quartile range; N: number.

$* P<0.05$, statistically different 
Table 5. Health economics data of all patients enrolled

\begin{tabular}{lccc}
\hline \multicolumn{1}{c}{ Health economics data } & PCG(N=68) & CG $(\mathrm{N}=41)$ & $P$ \\
\hline Medicine & $\mathrm{M}(\mathrm{IQR})$ & $\mathrm{M}(\mathrm{IQR})$ & \\
Examination & $2.51(1.79-3.27)$ & $2.89(2.29-4.30)$ & $0.002^{*}$ \\
Laboratory test & $0.05(0.03-0.09)$ & $0.06(0.05-0.08)$ & 0.051 \\
Treatment & $0.83(0.68-1.06)$ & $1.00(0.76-1.18)$ & 0.078 \\
Surgery & $7.99(6.59-9.66)$ & $9.00(7.59-11.09)$ & $0.024^{*}$ \\
Anesthesia & $0.46(0.45-0.47)$ & $0.47(0.40-0.47)$ & 0.527 \\
Consumables & $0.20(0.18-0.23)$ & $0.22(0.19-0.23)$ & 0.131 \\
Others & $3.94(3.55-4.50)$ & $4.45(3.45-5.45)$ & 0.140 \\
Total costs & $0.26(0.21-0.34)$ & $0.47(0.36-0.54)$ & $0.000^{*}$ \\
\hline
\end{tabular}

PCG: pre-COVID-19 group; CG: COVID-19 group; M: median; IQR: inter-quartile range.

$* P<0.05$, statistically different 


\section{Figure legend}

Figure1. Flow diagram of patient inclusion. 


\section{Figures}

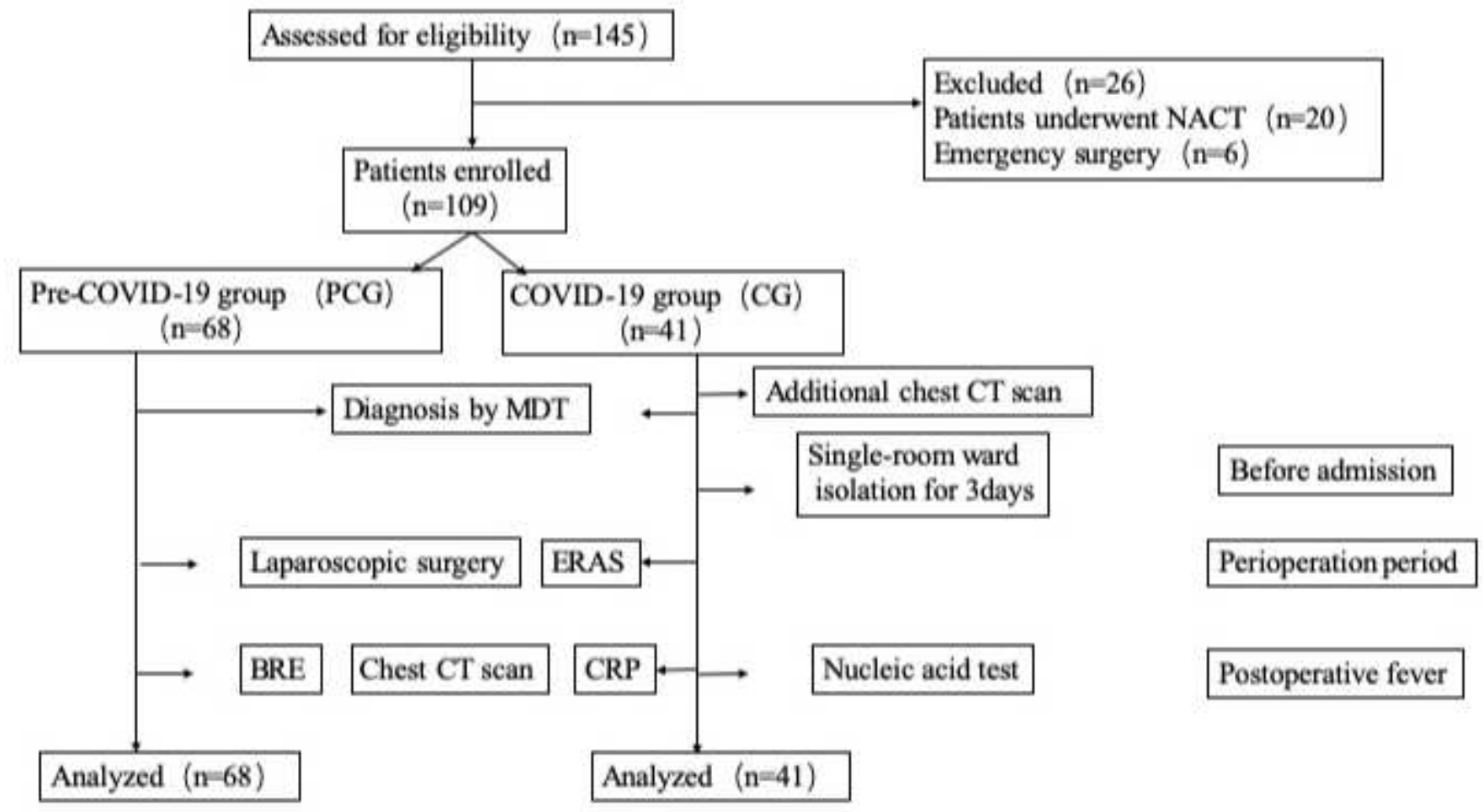

Figure 1

Flow diagram of patient inclusion. 